\section{USING ANALYTICAL MODELS TO CALIBRATE A DUAL-ROUNDABOUT INTERSECTION IN MICROSIMULATION}

Nikola Mitrovic, Ph.D. T.E.

Florida Atlantic University, Boca Raton, Florida, USA

Igor Dakic, Ph.D. candidate

Swiss Federal Institute of Technology (ETH), Zurich, Switzerland

Aleksandar Stevanovic, Ph.D. T.E.

Florida Atlantic University, Boca Raton, Florida, USA

DOI: 10.31075/PIS.64.02.02

Professional paper

Abstract: Calibration of roundabout microsimulation models is a challenging task that often relies on various field-related data that might not be always available. The calibration process of dual roundabouts might be even more demanding due to their rare presence in the field and literature. This paper proposes a straightforward but novel approach toward the calibration of dual roundabouts. The proposed method heavily relies on suggestions provided in the National Cooperative Highway Research Program (NCHRP) report 572 and easilyavailable information from the field (such as geometry and volume data). NCHRP is utilized to assess the key calibration parameters and corresponding capacity of a dual-roundabout model. The model validation is based on matching the capacity produced by the simulation model with the estimated capacity as it is proposed in NCHRP report. The numerical results of a single lane dual roundabout case study reveal the close match between capacities obtained from microsimulation tool (VISSIM) and NCHPR report. Moreover, the proposed approach can benefit traffic practitioners and planners dealing with the microsimulation models of roundabouts and limited data sets.

Keywords: roundabout, roundabout capacity, VISSIM calibration, NCHRP.

\section{INTRODUCTION}

A widely present tendency of converting traditional intersections into roundabouts is often motivated by an increase in safety, comfort and efficient movements of vehicular traffic. This trend can be expected to continue in the future, especially at those sites that are restricted to motorized road users. The evaluation of operational performance of traditional and roundabout intersections is required to assess the potential benefits and drawbacks of these two competitive intersection designs. Such an assessment, in order to properly cover aspects of efficiency and safety, often requires an accurately built (micro) simulation model.
This common practice relies on the presence of various types of field data that can be used for model calibration and validation purposes. For instance, studies [1] and [2] use extensive data sets collected from the field to perform a comprehensive calibration of the VISSIM models. However, this approach might not be possible for still un-built and rarely present types of roundabouts (e.g., dual roundabout), due to insufficient field information necessary for the calibration tasks.

Moreover, the traditional field-based calibration method might not always be feasible for existing roundabouts that are not properly covered with detectors. In such cases, the literature provides recommendations in the form of the Highway Capacity Manual (HCM) and National Cooperative Highway Research Program (NCHRP) reports, which can help practitioners to assess the key microsimulation parameters for known/given volumes and roadside information. Some of the NCHRP reports cover the most comprehensive roundabout dataset in the United States, which makes them suitable for calibration of many diverse roundabout types and sites [3]. In addition, $\mathrm{HCM}$ and NCHRP reports provide estimates of the roundabout capacities, which is valuable information for validating the microsimulation models [3]. An approach where simulation models are calibrated and validated based on relevant NCHRP and HCM reports can be beneficial when building, calibrating and validating miscrosimulation roundabouts models. In addition, the existing literature shows a lack of studies which consider calibration and validation of non-traditional types of roundabouts (such as dual roundabouts).

This study aims to fill the gaps in the existing body of knowledge by proposing a novel approach for calibrating a microsimulation model in the absence of the field data. In contrast to the traditional techniques [2], [4], which use various types of field data to calibrate a simulation model, this study follows the guidelines provided in the NHCRP report to calibrate the dual roundabout model by considering it as the two nearby traditional roundabouts.

A case study was conducted on a VISSIM microsimulation model of the proposed single-lane dual roundabout with a 30 -foot inscribed circle diameter in Palm Beach Gardens, Florida. This roundabout could potentially replace two existing close intersections in the future. The capacityrelated results from VISSIM were compared against the recommended outputs of the NHCRP report that was explicitly used to calibrate the model. The calibrated model is further used to evaluate the mobility and safety-related performance of dualroundabout for existing and projected AM peak volumes.

The rest of this paper is organized as follows. First, a brief overview of the relevant research is presented. Then, the authors explain how the 
VISSIM model is calibrated using the NHCRP report, and discuss the two most common applications of the calibrated roundabout model. In the subsequent section, the calibration and evaluations results are presented. The final section concludes the research paper and provides relevant ideas for future work.

\section{LITERATURE REVIEW}

There is an extensive literature on calibration of microsimulation models for roundabouts. Most of these studies rely on the VISSIM simulation platform [5] while the rest use other available tools (such as Sidra [6] and cellular automata models [1]). The VISSIM-related studies mostly focus on three key parameters, including: 1) Priority Rules or Conflict Areas that define the operational logic within roundabout and control the critical gap acceptance behavior; 2) Reduced Speed Areas, that provide speed control for circulating and inbound traffic; 3) Wiedeman 74 and Wiedeman 99 car following models that emulate driver and car following behaviors [2], [4].

Studies [2] and [4] present the most comprehensive work regarding the VISSIM model calibration. Wei et al. assessed the impact of various VISSIM parameters on the simulated roundabout capacity [4]. The authors proposed three calibration strategies to match the simulation capacity with HCM 2010. In another study, Li et al. used a large data set to perform a quantitative sensitivity analysis of the key parameters that can facilitate the modeling of roundabouts [2]. The authors deliver valuable calibration guidelines, and infer that the entire roundabout approach can be approximately divided into four speed zones. More importantly, the authors revealed that the use of priority rules often leads to a more consistent gap acceptance behavior than in the case of Conflict Areas (CA). Although the studies [2] and [4] provide valuable guidelines to practitioners, they heavily rely on diverse field datasets that is not always available.

There are only a few studies that explicitly use NCHRP reports in conjunction with the VISSIM microsimulation tool. Valdez et al. investigated control delay and level of service (LOS) for unbalanced approach volumes using a two-lane roundabout from NCHRP Report [7]. Moreover, Cicu et al. tried to find proper parameter estimates including critical gap and speed for a two-lane roundabout [8]. The authors also compared the modeled capacity with the capacity curve recommended by NCHRP Report 572.

The results from this study show that simulated capacity closely matches the corresponding estimates of NCHRP report, except in cases when the percentage of circulating traffic is extremely low or extremely high.
The calibrated roundabouts models are frequently utilized to explore the efficiency and safety aspects of different kinds of roundabout models. The efficiency is often studied in terms of vehicular delay and number of stops, while the safety aspect is usually investigated in terms of the number and types of potential conflicts. Most of the safety-related studies rely on the Surrogate Safety Assessment Model (SSAM), which uses the trajectories of vehicles to assess the safety surrogate measures [9], [10]. SSAM is a tool developed by Federal Highway Administration to estimate the number and type of conflict points based on vehicular trajectories [11]. SSAM defines the type of conflicts in one of the following categories: rear-end, crossing, and lanechanging conflicts based on the angle of movement of two conflicting vehicles when they are closest to each other. Finally, SSAM is capable of accurately assessing the surrogate safety measures, such as Time-to-Collision (TTC) [11].

In summary, the estimates of roundabout simulation parameters are often based on considerable fieldcollected data, while only a few studies marginally incorporated the information from NCHRP. NCHRP provides an easy-to-apply reference guide for determining whether a roundabout can handle current and/or future demand in terms of capacity. Therefore, it would be crucial to explore how such a straightforward approach aligns with the frequently used simulation tool in the contexts of roundabout operations.

\section{METHODOLOGY}

This paper presents a novel but still relatively straightforward approach for calibrating a dual roundabout VISSIM model in cases when only limited data (e.g., traffic counts) are available. The proposed method relies on the recommendations provided by NCHRP reports in two contexts: (1) assessing the model calibration parameters; and (2) obtaining the reference points for model validation. The properly validated model is further used to assess the approach of delay-based Level of Service (LOS) and safety surrogate measures of the proposed intersection design. The LOS and commonly used measures of effectiveness (MOEs) are computed in a traditional way. The number and type of potential vehicular conflicts are assessed using Surrogate Safety Assessment Model (SSAM) [11].

\subsection{Case Study}

The scope of the proposed study encompasses two closely spaced intersections at Central Blvd and University Blvd in Palm Beach gardens (Figure 1). These two unsignalized intersections can potentially be replaced by a single-lane dual roundabout if traffic flow continues to increase. 
The VISSIM roundabout model is built to assess the performance of mobility- and safety-related operations. The model is fed with the existing (2013) volumes during the AM peak and calibrated according to the recommendations provided in NCHRP report. Once calibrated, the model with the inflated volumes resembling projected traffic conditions (2035) is evaluated for mobility and safety applications. In addition, two operational strategies are investigated for the existing and projected volumes.

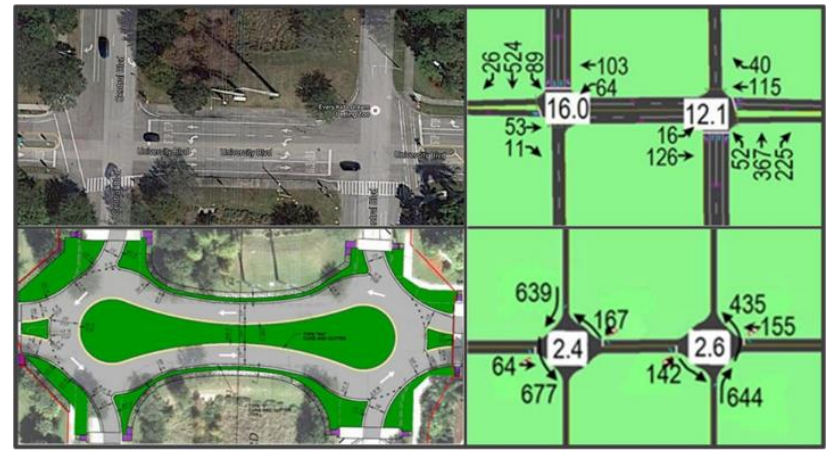

Figure 1. The geometry (left) and volumes (right) for the existing (top) and proposed (bottom) VISSIM model development

In the first strategy, which is referred to as 'traditional strategy,' the right of way is given to the vehicles circling the roundabout (Figure 2 top). In the second strategy, referred here as 'North-South ( $\mathrm{N}$ S)' operational strategy, the right of way is given to vehicles from Northbound and Southbound approaches (Figure 2 bottom).

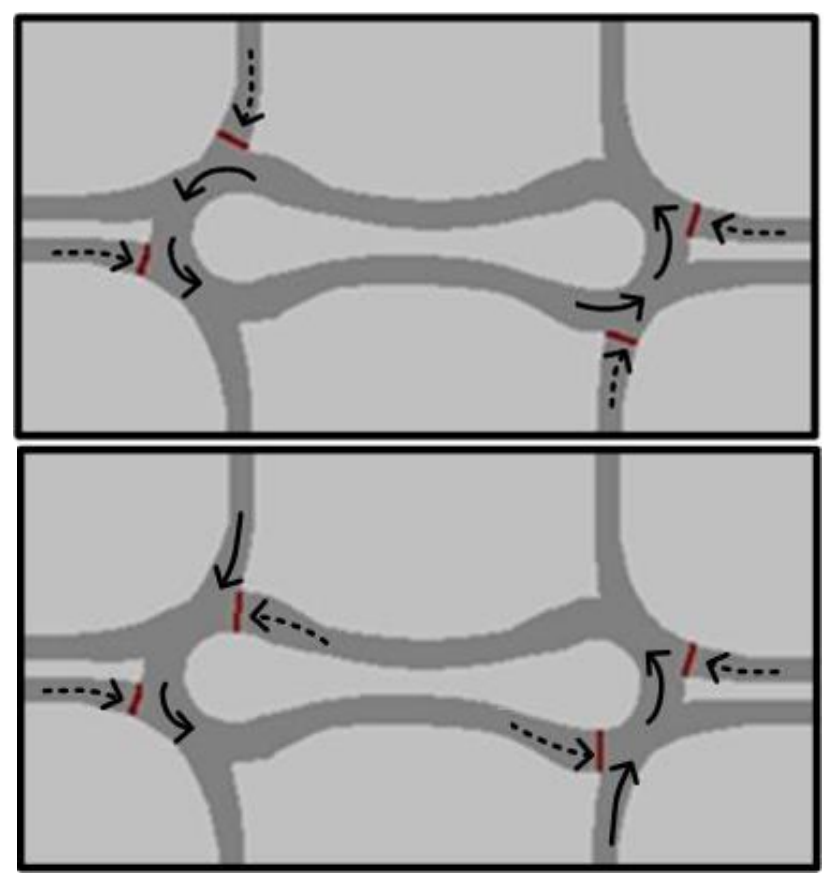

Figure 2. Evaluated operational strategies. The right way (see solid arrows) is given to the either circulating vehicles (top) or vehicles which are entering the roundabout from either north or south approach (bottom)

\subsection{Estimation of the VISSIM Parameters}

Since there is no explicit guidance for a dual roundabout in the NCHRP report, the study observes the site of interest as the two single, independent traditional roundabouts. Based on the geometry illustrated in Figure 1 (bottom left), the design speeds within the roundabout are determined using the recommendations from NCHRP report [3]:

$V=3.4415 \cdot R^{0.3861}$, for $e=+0.02$

$V=3.4614 \cdot R^{0.3673}$, for $e=-0.02$

where $R$ is a radius (ft) and $e$ is a superelevation $(\mathrm{ft} / \mathrm{ft})$. Eq. 1 is used to compute entry and exit speeds, while circulating speed is determined according to Eq. 2. The speeds defined in Eqs. 1 and 2 are further used to define the desired speed at approaches and within roundabout, respectively. Finally, the critical headway $\left(t_{c}\right)$ and follow-up headway $\left(t_{f}\right)$ are estimated using the following Eq. 3:

$t_{c}=2.67+\frac{3.95}{1+e^{b \cdot\left(d-d_{0}\right)}}$

$b=0.0001 \cdot D+0.0162 \cdot l_{p}+0.0028 \cdot l_{v c}$

$d_{0}=45.5-497.3 \cdot b$

$t_{f}=3.218-0.0001 \cdot v_{c}$

temp $=-1+\sqrt{(x-1)^{2}+\frac{8 \cdot x}{c \cdot T}}$

$d=\frac{3600}{c}+900 \cdot T \cdot \operatorname{temp}+\min \{x, 1\}$

where $d$ is the waiting time and $D$ is inscribed diameter $(\mathrm{m})$ of the roundabout. Indication variables $l_{v c}$ and $l_{p}$ refer to hourly pedestrian and circulating vehicular volumes, respectively. The variable $d_{0}$ is the waiting time at the deflection point of the logistic curve, $x=\frac{v}{c}$, where $v$ is the entry approach volume and $T$ is the period of analysis (h).

\subsection{Model validation}

The roundabout model is validated through a comparison of modeled and $\mathrm{HCM}$ capacity values. $\mathrm{HCM}$ methodology for single-lane roundabout estimates the capacity using the formula provided in NCHRP 572 [3]:

$c=\frac{3600}{t_{f}} \cdot e^{-\left(t_{c}-\frac{t_{f}}{2}\right) \frac{v_{c}}{3600}}$ 
where $v_{c}$ is the circulating volume in the roundabout and $c$ is estimated capacity. The gap times in the simulation model (approximated as the critical headway in Eq. 3) were slightly adjusted to provide a satisfactory matching (within 10\%) between the simulated and estimated capacities, which are proposed in the NCHRP report. This fine-tuning process is performed manually by adjusting gap times within priority rule (PR) settings. The Special Evaluation File in VISSIM is used to produce the discharge rates (and equivalent capacities) for all approaches. In order to acquire a discharge rate, mock 'signal heads' are placed at the positions where vehicles need to give the right of way, along with the data collection points (Figure 2). One should note that these 'signal heads' always display green light and had no impact on vehicular operations. Instead, they are only used to assess discharge rates of the relevant traffic flows. This approach is in alignment with the methodology of other studies that also use gap time as the calibration parameter to match the capacity obtained from simulation with the field-observed capacity [12].

\subsection{Applications of the roundabout model}

The roundabout miscrosimulation models are often used for mobility- and safety-related assessments. In this context, the scope of the study is restricted to the estimation of the key MOEs and the evaluation of the potential conflicts within the proposed, singlelane dual roundabout. Both performance aspects are investigated for the existing (2013) and projected (2035) AM peak volumes and both operational strategies.

\subsubsection{Level of Service (LOS) and Measure of Effectiveness (MOE)}

The LOS is computed for each roundabout approach according to HCM recommendations, where the LOS for roundabouts is estimated by using the same methodology is used for stop-controlled intersections. In other words, the LOS of a roundabout approach is determined by comparing computed (or measured) control delay with the proposed LOS thresholds (3). Since VISSIM does not generate the control delay as a direct output, authors used average total delay and average stopped delay to assess LOS. Average stopped delay and average total delay are used as lower and upper bound, respectively, in an estimation of control delay and LOS.

\subsubsection{Evaluation of Vehicular Conflicts}

As mentioned previously, one of the unique contributions of this research is the safety evaluation analysis of the dual roundabouts in the field-like microsimulation environment.
Second-by-second vehicular trajectories of all vehicles are recorded using special evaluation type in VISSIM, and later post-processed in SSAM.

\subsection{Model Calibration}

The input model parameters including desired speed, and critical and follow-up headways are defined using the recommendations given in NCHRP reports. Only the traffic demand is obtained (and further projected), while the other parameters in VISSIM roundabout model are approximated by their numerical default values. The critical headway is slightly tuned to resemble the expected capacity, as determined in NCHRP.
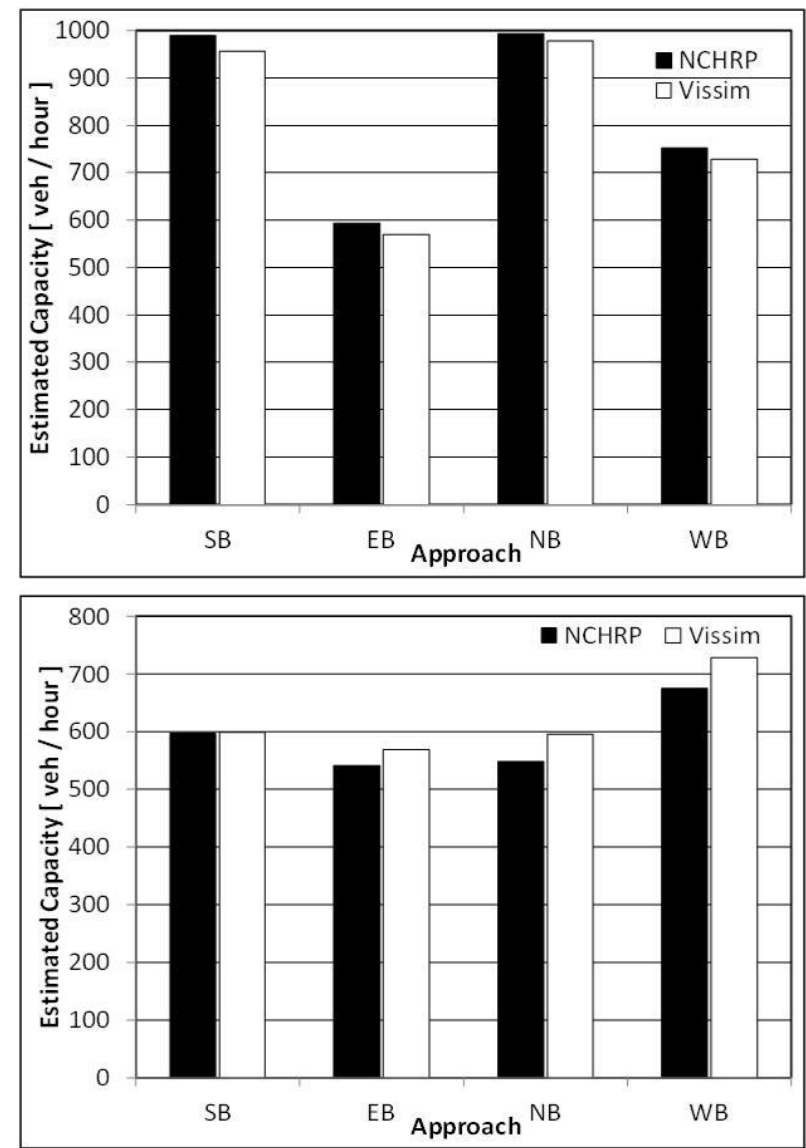

Figure 3. Estimated capacity of each roundabout approach according to traditional (top) and North-South operational strategy (bottom)

Figures 3 and 4 show the numerical values of estimated and modeled capacities, and the corresponding discharge rates, respectively, for each approach and operational strategy. The results from Figure 3 indicate close matches (values are within $\pm 10 \%$ ) between estimated and modeled capacities for both operational strategies. Intuitively, the effects of identical performance can be seen through discharge rates, considering that they are inversely proportional to the corresponding capacity (see Figure 4). 
More importantly, Table 1 shows there is close match between approximated and tuned critical headways. One can infer that NCHRP provides an effective approximation of the critical headway (see Table 1), which can be used as the initial value for model calibration purposes.

\section{RESULTS}

Ten VISSIM simulation runs were executed with random seeds for both traditional and N-S operational strategies using existing (2013) and projected (2035) AM peak volumes. Each simulation lasted for 75 minutes, or 1 hour of evaluation time and 15 minutes to warm-up.
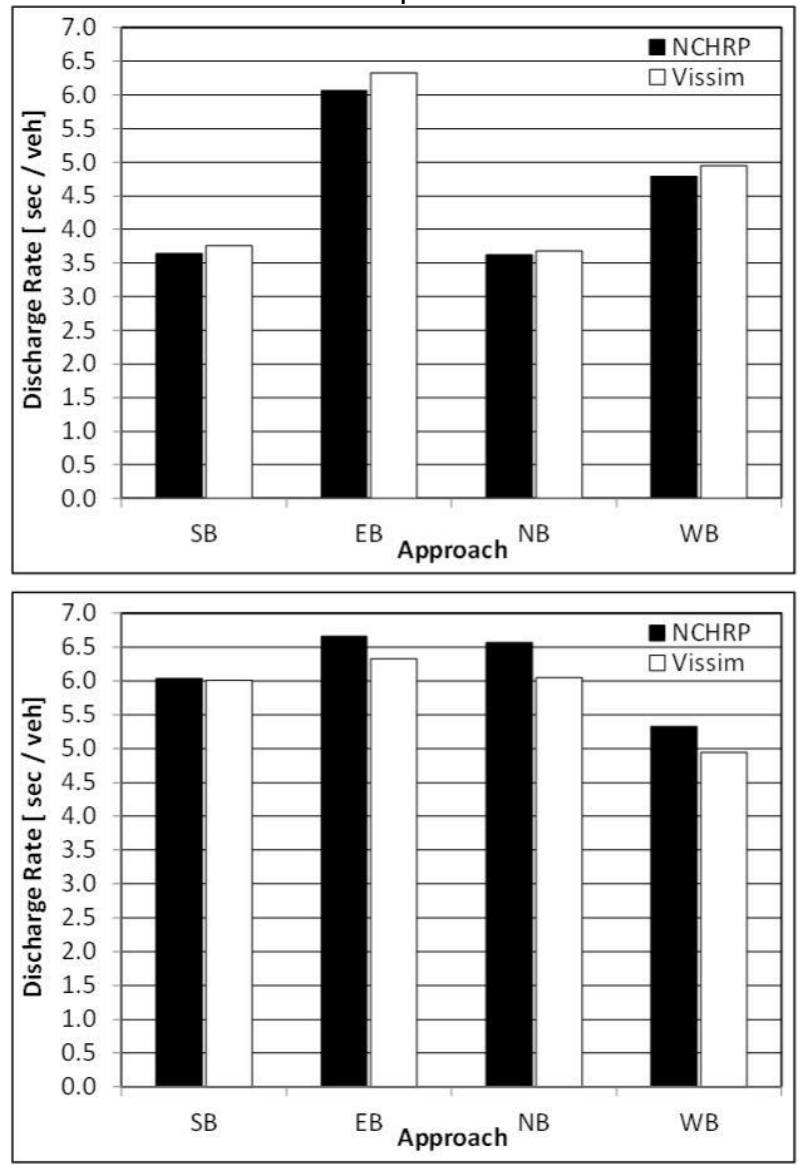

Figure 4. Estimated discharge rate of each roundabout approach according to traditional (top) and N-S operational strategy (bottom)

Table 1. Proposed (NCHRP) and tuned (VISSIM) critical headways (in seconds)

\begin{tabular}{|c|c|c|c|c|}
\hline $\begin{array}{c}\text { Operat. } \\
\text { Strategy }\end{array}$ & \multicolumn{2}{|c|}{ Traditional } & \multicolumn{2}{c|}{ North-South } \\
\hline Approach & $\begin{array}{c}\text { Analytical } \\
\text { (NCHRP) }\end{array}$ & $\begin{array}{c}\text { Simulation } \\
\text { Vissim) }\end{array}$ & $\begin{array}{c}\text { Analytical } \\
\text { (NCHRP) }\end{array}$ & $\begin{array}{c}\text { Simulation } \\
\text { (Vissim) }\end{array}$ \\
\hline SB & 5.08 & 5.00 & 5.21 & 5.40 \\
\hline EB & 5.28 & 5.40 & 5.28 & 5.00 \\
\hline NB & 5.09 & 5.00 & 5.22 & 5.10 \\
\hline WB & 5.26 & 5.40 & 5.26 & 5.00 \\
\hline
\end{tabular}

\subsection{Level of Service}

The properly calibrated VISSIM model is used to assess the performance of two operational strategies considered in the study. Table 2 shows the average stopped and total delay during AM periods for the existing and projected traffic. The stopped delay is explicitly recorded by VISSIM. On the other hand, the total delay is defined as the time difference between experienced and minimum (ideal) travel time through the intersection. Ideal travel time is calculated as a sum of link-wise travel times of these segments that lie along the considered route. Link-wise travel time is assessed using the (fixed) length of the particular link and corresponding desired speed (e.g., speed limit).

Table 2. Estimated average stopped delay (top) and total delay (bottom), both in seconds of the existing (2013) and projected (2035) traffic

\begin{tabular}{|c|c|c|c|c|}
\hline MOE & \multicolumn{4}{|c|}{ Average Stopped Delay } \\
\hline Demand & \multicolumn{2}{|c|}{ Current } & \multicolumn{2}{|c|}{ Future } \\
\hline $\begin{array}{c}\text { Operational } \\
\text { Strategy }\end{array}$ & Trad. & N-S & Trad. & N-S \\
\hline Southbound & 0.42 & 0.00 & 1.52 & * \\
\hline Eastbound & 13.52 & 13.92 & 33.95 & * \\
\hline Northbound & 0.32 & 0.00 & 0.90 & * \\
\hline Westbound & 4.36 & 2.99 & 12.72 & * \\
\hline MOE & \multicolumn{4}{|c|}{ Total Delay } \\
\hline Demand & \multicolumn{2}{|c|}{ Current } & \multicolumn{2}{|c|}{ Future } \\
\hline $\begin{array}{c}\text { Operational } \\
\text { Strategy }\end{array}$ & Trad. & N-S & Trad. & N-S \\
\hline Southbound & 1.53 & 0.00 & 6.25 & * \\
\hline Eastbound & 17.89 & 18.38 & 41.79 & * \\
\hline Northbound & 0.42 & 0.00 & 1.02 & * \\
\hline Westbound & 8.01 & 6.11 & 20.08 & * \\
\hline
\end{tabular}

From Table 2, it can be observed that certain approaches show very poor performance. LOSs with asterisks denote cases when VISSIM was not capable of producing the output performance due to very high demand (such as the 2035 scenarios) and simulated priority strategy (N-S operational strategy). In that case, a gridlock situation was created which did not allow VISSIM to accurately estimate delays. Table 3 shows the corresponding LOS for each evaluated scenario, which is derived from computed approach delays. To summarize, the results show that both operational strategies would work with equal efficiency in current traffic conditions. However, once the traffic demand reaches 2035 levels, only the traditional strategy, which gives priority to the vehicles within roundabout, can produce an efficiently sustainable solution. 
Table 3. Estimated Level of Service (LOS) for the existing (2013) and projected (2035) traffic conditions

\begin{tabular}{|c|c|c|c|c|}
\hline Demand & \multicolumn{2}{|c|}{ Current } & \multicolumn{2}{c|}{ Future } \\
\hline $\begin{array}{c}\text { Operational } \\
\text { Strategy }\end{array}$ & Trad. & N-S & Trad. & N-S \\
\hline Southbound & $\mathrm{A}$ & $\mathrm{A}$ & $\mathrm{A}$ & $\mathrm{F}^{*}$ \\
\hline Eastbound & $\mathrm{C}(\mathrm{B})$ & $\mathrm{C}(\mathrm{B})$ & $\mathrm{E}(\mathrm{D})$ & $\mathrm{F}^{*}$ \\
\hline Northbound & $\mathrm{A}$ & $\mathrm{A}$ & $\mathrm{A}$ & $\mathrm{F}^{*}$ \\
\hline Westbound & $\mathrm{A}$ & $\mathrm{A}$ & $\mathrm{C}(\mathrm{B})$ & $\mathrm{F}^{*}$ \\
\hline
\end{tabular}

\subsection{Vehicular Conflicts}

The safety evaluation analysis of two proposed operational strategies is performed for existing and projected demand during the AM peak period. The SSAM tool uses second-by-second vehicular trajectories collected by VISSIM to assess the number and types of potential conflict points. Table 4 summarizes the number of conflicts occurring on the case-study network. Two conflict types are detected: rear-end and lane-change conflicts. Please note that lane changing conflicts in the context of single-lane dual roundabout might only appear at entering and exiting points of the roundabout. In addition, the results of SSAM analysis show that the SSAM does not recognize any crossing conflicts, which is a logical outcome given that the roundabouts eliminate crossing conflicts.

Table 4 shows that the N-S operational strategy generates significantly lowers the number of conflicts than the traditional strategy. Moreover, only a minimal number of lane-change conflicts was detected. As it can be observed from Table 4, the most numerous conflicts are rear-end at more than $95 \%$ of all estimated conflicts points. One should also note that the total number of conflicts does not necessarily transfer into an equivalent number of potential crashes, as some researchers question the usefulness of conflict analysis in estimating the impact of various operational scenarios on the safety of certain intersection designs. Finally, Table 4 clearly shows that the total number of conflicts increases with an increase in traffic demand, while Figures 5 and 6 illustrates the specific spatial distribution of different conflict types for the different traffic demand and operational strategies.

Table 4. Estimated number and type of conflicts for the existing (2013) and projected (2035) traffic

\begin{tabular}{|c|c|c|c|c|c|}
\hline \multirow{2}{*}{\multicolumn{2}{|c|}{$\begin{array}{c}\text { Demand } \\
\text { Operational } \\
\text { Strategy }\end{array}$}} & \multicolumn{2}{|c|}{ Current } & \multicolumn{2}{|c|}{ Future } \\
\hline & & \multirow{2}{*}{$\begin{array}{r}\text { Trad. } \\
73.0\end{array}$} & \multirow{2}{*}{$\begin{array}{l}\text { N-S } \\
25.1\end{array}$} & \multirow{2}{*}{$\begin{array}{l}\text { Trad. } \\
310.8\end{array}$} & \multirow{2}{*}{$\begin{array}{c}\text { N-S } \\
\text { * }\end{array}$} \\
\hline \multirow{3}{*}{ 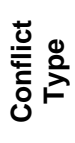 } & Rear-End & & & & \\
\hline & Lane-Change & 1.0 & 0.4 & 1.7 & * \\
\hline & Total & 74.0 & 25.5 & 312.5 & * \\
\hline
\end{tabular}

Interestingly, under the current traffic demand, the $\mathrm{N}-\mathrm{S}$ operational strategy generates much fewer conflicts than the traditional strategy. However, in 2035 traffic it is estimated that the N-S strategy would create a gridlock which makes the estimation of traffic conflicts irrelevant, as non-moving vehicles may not create conflicts, but the trips are not being executed.

\section{CONCLUSION AND FUTURE DIRECTIONS}

This study investigated a possibility to calibrate and validate the VISSIM model of dual roundabout concept by following the procedure given in NCHRP Report 572. In contrast to the traditional approach that uses various field-related data, the proposed method only relies on easily available geometric and volume information. The results reveal that NCHRP Report 572 and VISSIM produced similar output results (within 10\%) for the identical input parameters. The calibrated VISSIM model is further used to assess efficiency and safety of the dual roundabout intersection. Two different strategies are evaluated and compared for both the existing and projected traffic. The traditional strategy has been proven to be better in terms of the efficiency than the $\mathrm{N}-\mathrm{S}$ operational strategy. In terms of the estimated conflicts it seems that N-S strategy generates fewer conflicts in current situation but it causes a severe gridlock for future traffic demand and therefore it deems inappropriate for the given location.

In general, it can be concluded that the NCHRP Report 572 is a useful source for development of planning-related and other models when only a limited field data are available. Recommendations provided in this report allow researchers to perform mobility- and safety-related applications, even in the case of insufficient traffic information. Finally, future research should investigate similar procedures and apply them to other types of roundabouts. In addition, it still remains uninvestigated whether similar results can be obtained for different combination of entering and circulating traffic in the context of dual roundabouts. 


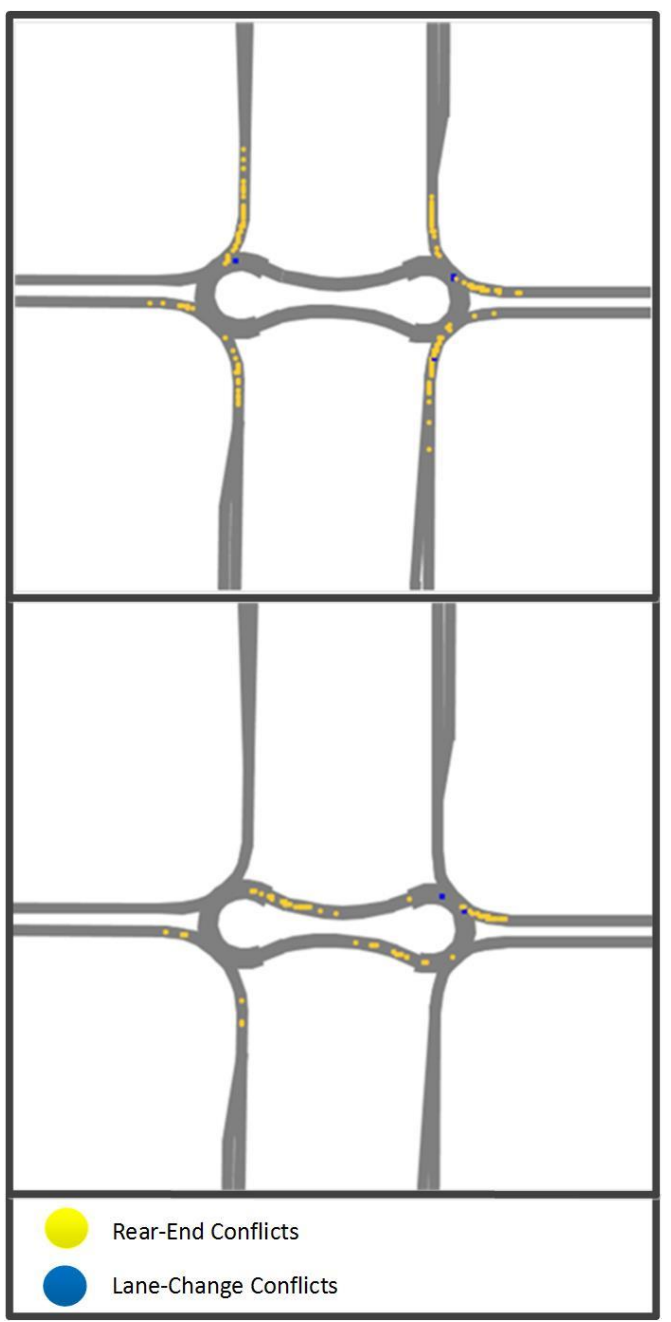

Figure 5. Spatial location of the possible vehicular conflict for the existing traffic in the case of traditional (top) and North-South (bottom) operational strategies

\section{References}

[1] Belz, N. P., Hall L. A., Montague J. (2016). Influence of priority taking and abstaining at single-lane roundabouts using cellular automata. Transportation Research Part C: Emerging Technologies. Vol 69, pp. 134-149.

[2] Li, Z., DeAmico M., Chitturi M. V., Bill A. R., Noyce D. A. (2013). Calibration of VISSIM Roundabout Model: A Critical Gap and Follow-up Headway. Transportation Research Board 92nd Annual Meeting.

[3] Rodegerdts, L.(2007). Roundabouts in the United States. Vol. 572. Transportation Research Board.

[4] Wei, T., Shah H. R., Ambadipudi R.(2012). VISSIM Calibration for Modeling Single-Lane Roundabouts: CapacityBased Strategies. Transportation Research Board 91st Annual Meeting.

[5] Arroju, R., Gaddam H. K., Vanumu L. D., Rao K. R. (2015). Comparative evaluation of roundabout capacities under heterogeneous traffic conditions. Journal of Modern Transportation, pp. 310-324.

[6] Muley, D., Al-Mandhar H. S. (2014). Performance Evaluation of Al Jame'Roundabout Using SIDRA. World Academy of Science, Engineering and Technology, International Journal of Civil, Environmental, Structural, Construction and

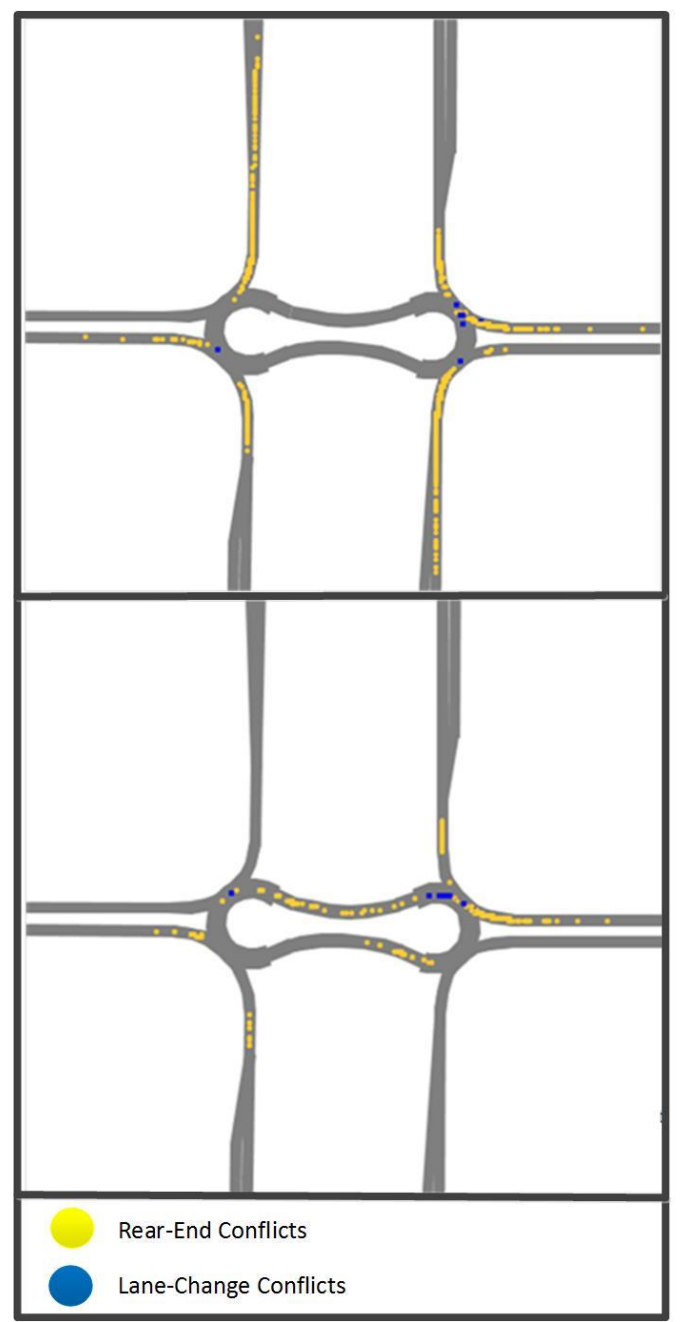

Figure 6. Spatial location of the possible vehicular conflict for the projected traffic in the case of traditional (top) and North-South (bottom) operational strategies

Architectural Engineering, pp. 1291-1296.

[7] Valdez, M., Cheu R. L., Duran, A. C. (2011). Operations of modern roundabout with unbalanced approach volumes. Transportation Research Record: Journal of the Transportation Research Board, no. 2265, pp. 234-243.

[8] Cicu, F., Illotta P. F., Bared J., Isebrands H.(2011) VISSIM Calibration of Roundabout Traffic Performance. Transportation Research Board 90th Annual Meeting.

[9] Al-Ghandour, M. N., Schroeder B. J., Williams B. M., Rasdorf W. J. (2011). Conflict models for single-lane roundabout slip lanes from microsimulation: Development and validation. Transportation Research Record: Journal of the Transportation Research Board, no. 2236, pp. 92-101.

[10]Vasconcelos, L., Neto L., Seco Á. M., Silva A. B. (2014) Validation of the Surrogate Safety Assessment Model for Assessment of Intersection Safety. Transportation Research Record: Journal of the Transportation Research Board, no. 2432, pp. 1-9.

[11]Gettman, D., Pu, L., Sayed, T., Shelby, S. G. (2008). Surrogate safety assessment model and validation: Final report (No. FHWA-HRT-08-051).

[12]Zheng, D., Chittur M. V., Bill A. R., Noyce D. A (2012). Critical Gaps and Follow-up Headways at Congested Roundabouts. Transportation Research Board 91st Annual Meeting. 